\title{
Expression Sites of Neural Stem Cell-Related Genes in the Monkey Retina
}

Maho Shibata $^{1}$, Tsunehiko Ikeda ${ }^{1 *}$, Kimitoshi Nakamura ${ }^{2}$, Keigo Kakurai $^{1}$, Seita Morishita ${ }^{1}$, Masanori Fukumoto ${ }^{1}$, Teruyo Kida $^{1}$, Taeko Horie $^{1}$ and Hidehiro Oku ${ }^{1}$

${ }^{1}$ Department of Ophthalmology, Osaka Medical College, Takatsuki City, Osaka, Japan

${ }^{2}$ Nakamura Eye Clinic, Matsumoto, Nagano, Japan

\begin{abstract}
Objectives: Based on the hypothesis that undifferentiated retinal stem cell (RSC)-like cells exist in the fovea (the light-stressed, concave, avascular center of the retina where light is focused), we investigated the expression sites of neural stem cell (NSC)-related genes in the monkey retina.
\end{abstract}

Methods: Cynomolgus monkeys were euthanized, and both eyes were then enucleated. Each eye was hemisected near the limbus, and flat-mounted retina samples were then prepared. Using a stereomicroscope, 1-mm $x 1-\mathrm{mm}$ blocks of the retina at the fovea, mid-periphery, and extreme periphery were then excised. These samples were used for real-time polymerase chain reaction analysis of the NSC-related gene (nestin, PAX6, and SOX2) expression at each site.

Results: Nestin expression was high in the fovea, with a lower expression in the mid-periphery and extreme periphery. No differences in PAX6 gene expression were found in the fovea, mid-periphery, and extreme periphery. SOX2 expression was highest in the extreme periphery, with decreased expression in the mid-periphery and fovea.

Conclusions: Our finding that nestin expression was highest in the fovea suggests that foveal retinal cells may have more undifferentiated characteristics that are different from retinal cells at other sites.

Keywords: Retinal stem cells; Nestin; PAX6; SOX2; Real-time PCR; Fovea

\section{Introduction}

The existence of stem cells in the central nervous system (CNS) has been reported, and their use in regenerative medicine has recently attracted interest $[1,2]$. Retinal stem cells (RSCs), with the ability to differentiate into neurons, glia, and photoreceptors, have also been reported at the boundary between the retina and pars plana, which is the so-called ciliary marginal zone [3-6]. Stem cells have also been found to exist at sites other than tissue boundaries, such as in recessed areas like small intestinal crypts, hair follicles, and the dermis of the skin $[7,8]$. In addition, stem cells often exist at avascular sites such as the corneal limbus where corneal epithelial stem cells reside [9]. It is our belief that undifferentiated RSC-like cells may also exist in the fovea, which has a combination of these anatomical characteristics.

In a previous study using tissue sections obtained from monkey eyes, we found strong positive staining in the fovea for the neural stem cell (NSC) marker nestin, and discussed its possible involvement in the onset of idiopathic macular holes [10]. In the present study, we also used monkey eyes to analyze the gene expression of the neural stem cell (NSC) markers nestin, PAX6, and SOX2 at different sites in the retina using real-time polymerase chain reaction (PCR).

\section{Methods}

This study involved the use of two, healthy, male cynomolgus monkeys (body weight range: 11-14 kg). Both animals were used in accordance with the ARVO Statement for the Use of Animals in Ophthalmic and Vision Research.

\section{Excision of monkey retina and RNA extraction}

For the excision of the retina and RNA extraction, the monkeys were first euthanized, and both eyes were then enucleated. Next, each eye was hemisected near the limbus and eye cups were prepared by removing the cornea, iris, lens, and vitreous. Flat-mounted retina samples were then prepared. Using a stereomicroscope, $1 \mathrm{~mm} \times 1 \mathrm{~mm}$ blocks of the retina at the fovea, mid-periphery, and extreme periphery were excised. To prevent further RNase action and to stabilize the intracellular RNA, the samples were immersed in RNAlater ${ }^{B}$ (QIAGEN, Valencia, CA, USA) stabilization reagent and the RNA was then extracted. The samples were homogenized in lysis buffer, and the RNA was extracted with the RNeasy Plus Mini Kit (QIAGEN). The RNA concentrations and purity were calculated from the absorbance at $260 / 280 \mathrm{~nm}$.

\section{Primer design and synthesis}

Primers for the real-time PCR were designed using the algorithms of a primer design system [Perfect Real Time support system; Takara Bio Inc., Otsu, Shiga, Japan (http://www.takara-bio.co.jp/prt/intro. htm)] for the target genes nestin, PAX6, and SOX2, and for the reference gene GAPDH (Table 1).

\section{Synthesis of cDNA}

For each sample after pretreatment, cDNA was synthesized using a Prime Script ${ }^{\circledR}$ RT Reagent Kit (Perfect Real Time, Code: RR037A; Takara Bio)

*Corresponding author: Tsunehiko Ikeda, MD, Department of Ophthalmology, Osaka Medical College, 2-7, Daigaku-machi, Takatsuki City, Osaka 569-8686, Japan, Tel: +81-72-684-6434; Fax: +81-72-682-0995; E-mail: tikeda@poh.osaka-med.ac.jp

Received November 03, 2015; Accepted December 08, 2015; Published December 10, 2015

Citation: Shibata M, Ikeda T, Nakamura K, Kakurai K, Morishita S, et al. (2015) Expression Sites of Neural Stem Cell-Related Genes in the Monkey Retina. J Stem Cell Res Ther 5: 319. doi:10.4172/2157-7633.1000319

Copyright: ( 2015 Shibata M, et al. This is an open-access article distributed under the terms of the Creative Commons Attribution License, which permits unrestricted use, distribution, and reproduction in any medium, provided the original author and source are credited. 


\begin{tabular}{|c|c|c|}
\hline Target Name & $\begin{array}{c}\text { amplification site } \\
(\text { (mRNA) }\end{array}$ & 5'-base sequence-3' \\
\hline \multirow{2}{*}{ GAPDH } & & GAATGCCTTCATGGTGTGGTC \\
\hline & $306 \quad 429$ & GTCTGCGAGCTGGTCATGGA \\
\hline \multirow{2}{*}{ Nestin } & & ССTGCTACCCTTGAGACACCTG \\
\hline & 8861027 & GGGCTCTGATCTCTGCATCTG \\
\hline \multirow{2}{*}{ PAX6 } & & AGATGAGGCTCAAATGCGACTTC \\
\hline & 398479 & GGCCTCAATTTGCTCTTGGGTA \\
\hline \multirow{2}{*}{ sox2 } & & ACAGTCCGGACCGCGTTAAG \\
\hline & 113226 & GCTTGCTGATCTCCGAGTTGTG \\
\hline
\end{tabular}

Table 1: Primer information

\section{Real-Time PCR and selection of samples for the calibration} curves

Using the synthesized cDNA as a template, real-time PCR reaction by the intercalator method for the target genes and reference gene was performed with SYBR' Premix Ex TaqTM II (Tli RNaseH Plus) (Code: RR820A; Takara Bio). The reactions were performed using a Thermal Cycler Dice Real Time System II (Code: TP900; Takara Bio), with the $\mathrm{Ct}$ values determined by the second derivative maximum (SDM) method. Based on the obtained results, samples for the calibration curves were selected from the samples that exhibited high expression for each gene.

\section{Real-Time PCR and preparation of calibration curves}

After serially diluting the samples for the calibration curves in 8 steps and performing the real-time PCR reaction, the calibration curves for each analyzed gene were prepared from the $\mathrm{Ct}$ values and dilution series.

\section{Measurement of $\mathrm{Ct}$ values for each sample}

Reactions ( $\mathrm{n}=2$ ) were performed for each sample using $10 \mathrm{ng}$ equivalents of the total RNA. The quantity (Qty(SDM)) for each gene was then calculated based on the Ct values and calibration curves.

\section{Calculation of relative quantities}

For each sample, the relative quantities [Rel. Qty(SDM)] of the target genes in each sample were calculated by normalizing the average Qty(SDM) [Qty Avg. (SDM)] of the target genes to the average Qty(SDM) of the reference gene. Multiplate RQ software (Takara Bio) was used to calculate the relative quantities.

\section{Results}

Graphs of the Rel.Qty(SDM) for the target genes in each sample, normalized for the Qty Avg. (SDM) of the reference gene in each sample, are shown in Figure 1 through 3. A high expression of nestin was found in the fovea, with a lower expression found in the midperiphery and extreme periphery (Figure 1). No differences in PAX6 gene expression were found in the fovea, mid-periphery, and extreme periphery (Figure 2). SOX2 expression was highest in the extreme periphery, with decreased expression in the mid-periphery and fovea (Figure 3).

\section{Discussion}

It had long been believed that neurons in the CNS of adult mammals cannot regenerate. However, neuron regeneration in areas such as the hippocampus dentate gyrus has recently been reported [1,2]. It is now known that newly regenerated neurons in the hippocampus form synapses with existing neurons, and function as part of the neural network. The hippocampus is closely associated with learning and memory, and is an area that is easily injured by stress. Therefore, a continual regeneration of neurons in the adult hippocampus may be associated with the repair of the injured CNS. It has become clear that at the site of these newly regenerated neurons in the hippocampus, radial glia and astrocytes, with radial projections into the cortex, mainly function as neural progenitor cells (NPCs) [11]. This finding has also attracted attention in the field of regenerative medicine.

In the retina, which is also part of the CNS, RSCs (which have the ability to differentiate into neurons, glia, and photoreceptors) have additionally been reported at the boundary between the retina and pars plana; i.e., the so-called ciliary marginal zone [3-6]. Stem cells have reportedly also been found in the corneal limbus at the boundary of the conjunctiva and cornea [9]. Thus, stem cells are often present at tissue junctions. Furthermore, stem cells have also been found to exist at sites other than tissue boundaries, such as in recessed areas like small

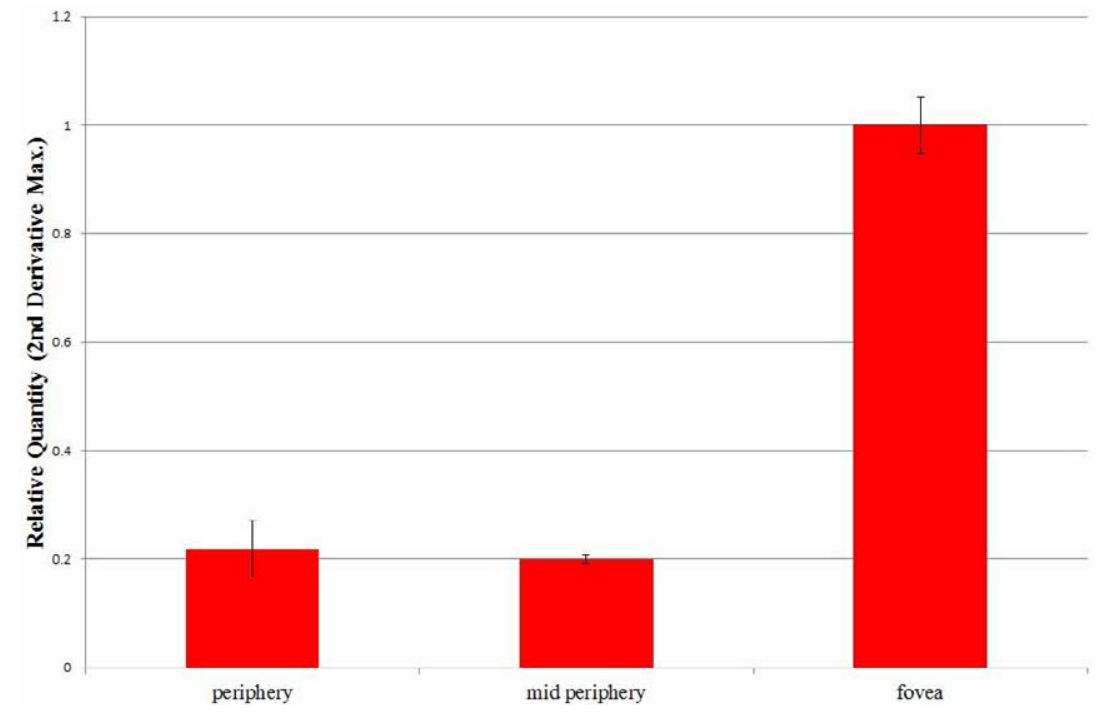

Figure 1: Graph of the relative quantity $2^{\text {nd }}$ derivative max [Rel.Qty(SDM)] of the target gene nestin. Reference gene: GAPDH. 


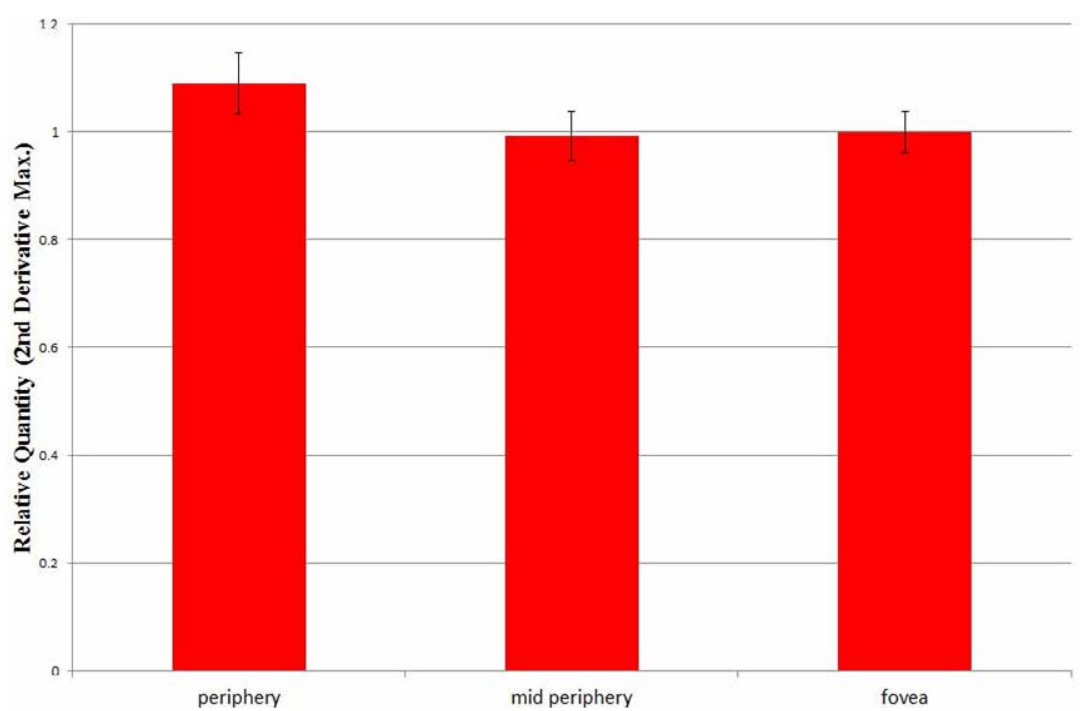

Figure 2: Graph of the Rel. Qty(SDM) of the target gene PAX6. Reference gene: GAPDH.

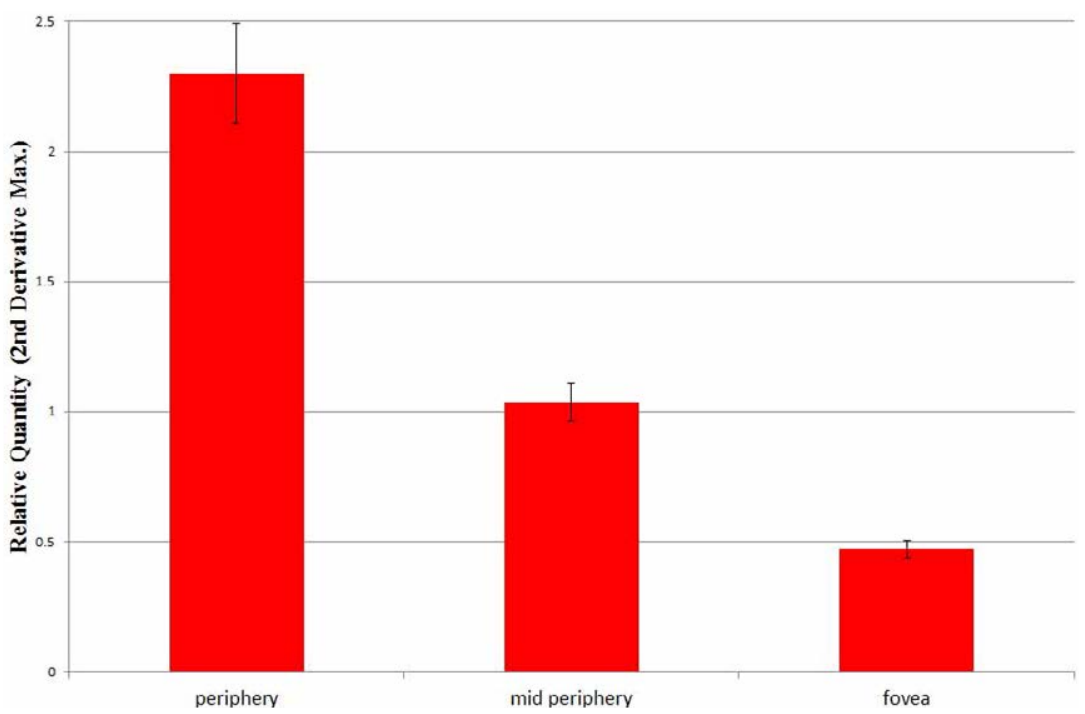

Figure 3: Graph of the Rel.Qty(SDM) of the target gene SOX2. Reference gene: GAPDH.

intestinal crypts, hair follicles, and the dermis of the skin [7,8]. They often tend to be found in avascular areas with low oxygen supply. These niches (recesses) are often hypoxic environments that are ideal for stem cells. The fovea shares many anatomical characteristics with these other tissues where stem cells reside. The fovea is a concave avascular area at the center of retina where light is focused, and it is continually lightstressed. Therefore, we theorize that the fovea may be a site of RSCs.

One clinical finding that supports our hypothesis is that there is a propensity for retinal diseases to involve the macula. Idiopathic epiretinal membrane is a disease with cell proliferation in the macula, and despite complete removal of the epiretinal membranes during a vitrectomy, recurrence of the membranes sometimes occurs postoperatively. This suggests that undifferentiated stem cells of the fovea differentiate and grow into glial cells. In fact, nestin-positive cells have been found in epiretinal membranes [12].
Moreover, during the postoperative course after vitrectomy for idiopathic macular holes, despite early improvement in the shape of the fovea as seen on funduscopy or optical coherence tomography (OCT) at the early postoperative stage, visual acuity continuously improves on a long-term basis. This suggests that undifferentiated RSCs of the fovea differentiate over time into neurons that form the sensory retina, with the visual function acquired later._Kishi et al. proposed that idiopathic macular holes are mainly caused by the vitreoretinal traction of vitreous gel that forms the posterior wall of a premacular vitreous cortex pocket [13]. However, much remains unknown about why macular holes concentrically enlarge, or why OCT shows findings of retinoschisis with macular holes. The same holds true for the question of why traumatic macular holes often spontaneously close.

In a previous study using vitreous samples obtained at vitrectomy, we reported increased activity of serine proteases such as chymase and 
tryptase in cases of idiopathic macular holes and idiopathic epiretinal membrane [14]. Moreover, chymase activity was predominantly elevated in cases of idiopathic macular holes, and tryptase activity was predominantly elevated in cases of idiopathic epiretinal membrane. These serine proteases are produced in mast cells. In the eye, however, while mast cells are found in many areas including the ciliary body, choroid, sclera, and conjunctiva, among others, they are not found in the retina [15].

It remains unknown why serine protease activity is increased in the vitreous in cases of idiopathic macular holes and idiopathic epiretinal membrane. However, tryptase is involved in tissue fibrosis, and chymase induces apoptosis of cells with relatively low differentiation, including vascular smooth muscle cells, bronchial smooth muscle cells, and cardiomyocytes [16-20]. In other words, if we presume that there are undifferentiated cells in the fovea, then with regard to the action of these serine proteases, idiopathic epiretinal membranes may develop when tryptase activity is predominant, and idiopathic macular holes may develop when chymase activity is predominant. In fact, OCT often shows findings of retinoschisis near the fovea in cases of idiopathic macular holes. This suggests that there may be some type of dysfunction in Müller cells, which support the sensory retina.

In a previous study, we performed immunostaining in cynomolgus monkey eyes for nestin, an NSC marker, in sections near the macula, mid-periphery, equator, and extreme periphery [10]. In that study, our comparison of nestin-positive cell density at each region clearly showed a higher density in the macula, but no significant differences among the other sites. Hematoxylin and eosin staining was also performed in monkey eyes intravitreally injected with chymase and in a sham control eye. No clear differences in the macula were seen between the sham control eye and the low-dose chymase-treated eye. However, findings in the high-dose chymase-treated eye suggested that there was thickening of the posterior hyaloid membrane in the macular region. TdT-mediated dUTP nick-end labeling (TUNEL) staining showed no positive cells in the sham control eye, but in the high-dose chymase-treated eye, scattered TUNEL-positive cells around the fovea were observed. Nestin is known as a marker for NSCs and RSCs. Thus, our findings suggested that undifferentiated cells with stem cell-like properties were present in the fovea.

Based on those previous results, we designed our present study to use real-time PCR to further analyze the expression of NSC-related genes at different regions of the monkey retina (i.e., the fovea, midperiphery, and extreme periphery). Similar to our previous findings in the tissue sections of the monkey eye, the present results showed high nestin expression in the fovea. However, no differences in PAX6 gene expression were found among the different regions.

PAX6 is involved in foveal formation, and its expression in the retinal pigment epithelium (RPE) induces transdifferentiation into retinal cells [21]. PAX6 may be highly expressed in the fovea early in development, but in adult monkeys, this difference is probably lost. The results for SOX2 were opposite to those for nestin. Namely, SOX2 gene expression was lowest in the fovea and highest in the periphery. SOX2 is known to be a transcription factor that regulates self-replication of RSCs. In the adult rat brain, SOX2 is reportedly expressed not only by NPCs, but also by astrocytes [22]. SOX2 expression has also been reported in glial cells such as astrocytes in the retina, but since the fovea is avascular with few astrocytes, SOX2 expression in the fovea is probably low.

When retinal injury occurs in adult fish and birds, Müller cells in the retina reportedly become like NPCs, proliferate, and differentiate to retinal neurons, or again to Müller cells [23]. It has long been thought that this type of retinal regeneration does not occur in mammals, but recently, Müller-cell-derived regeneration in the retina of adult mammals has also been reported [24,25]. Since all retinal layers were excised in this present study, it is unclear which cells in the sensory retina expressed nestin. In our previous study that examined immunostaining for nestin in monkey eyes, many nestin-positive cells were observed in the inner nuclear layer and adjacent fovea [10]. This suggests that nestin may be expressed by Müller cells in the sensory retina or by Müller cell cones in the fovea.

Nestin expression is known to increase if the retina is exposed to mechanical injury or hypoxia [26,27]. In adult autopsy eyes, Bhatia et al. performed immunostaining for expression of NSC markers in Müller cells in different regions of the retina [28]. They reported cells positive for both vimentin and nestin mainly in the inner nuclear layer, thus suggesting the likelihood of Müller cells. Many cells in the extreme periphery area of the retina were also co-positive for vimentin and nestin. They also reported that although the number of co-positive cells decreased towards the posterior pole, there were still a substantial number of co-positive Müller cells at the posterior pole. However, it should be noted that the examination performed in that study was not limited to only the area around the fovea and not focused fovea.

Nestin-positive Müller cells are known to exist in fetuses [29], and nestin-positive Müller cells exist throughout most areas of the retina in adults. The results of this current study demonstrated that the number of nestin-positive Müller cells was relatively higher in the fovea than at other areas of the retina. The macular region is clinically considered to have a special physiological function not found in other parts of the retina. In other words, differences in the distribution of nestin-positive cells within the retina may be involved in disease onset. However, there is insufficient evidence at the present time to presume that this means that RSCs exist. If indeed there are a large number of nestin-positive undifferentiated Müller cells in the macula,_then this may be related to the onset of many macular-specific diseases, including epiretinal membrane, macular holes, and macular edema._If further research studies can elucidate the pathogenesis of macular disease, then those findings could possibly lead to the development of novel treatments.

\section{Acknowledgements}

The authors wish to thank John Bush for reviewing the manuscript.

\section{References}

1. Alvarez-Buylla A, Lois C (1995) Neuronal stem cells in the brain of adult vertebrates. Stem Cells 13: 263-272. [PubMed]

2. Cayre M, Canoll P, Goldman JE (2009) Cell migration in the normal and pathological postnatal mammalian brain. Prog Neurobiol 88: 41-63. [PubMed]

3. Perron M, Harris WA (2000) Retinal stem cells in vertebrates. Bioessays 22: 685-688. [PubMed]

4. Tropepe V, Coles BL, Chiasson BJ, Horsford DJ, Elia AJ, et al. (2000) Retinal stem cells in the adult mammalian eye. Science 287: 2032-2036. [PubMed]

5. Kubota R, Hokoc JN, Moshiri A, McGuire C, Reh TA (2002) A comparative study of neurogenesis in the retinal ciliary marginal zone of homeothermic vertebrates. Brain Res Dev Brain Res 134: 31-41. [PubMed]

6. Reh TA, Fischer AJ (2001) Stem cells in the vertebrate retina. Brain Behav Evol 58: 296-305. [PubMed]

7. Potten CS (1992) The significance of spontaneous apoptosis and induced apoptosis in the gastrointestinal tract of mice. Cancer Metastasis Rev 11: 179195. [PubMed]

8. Ijiri K (1989) Apoptosis (cell death) induced in mouse bowel by 
Citation: Shibata M, Ikeda T, Nakamura K, Kakurai K, Morishita S, et al. (2015) Expression Sites of Neural Stem Cell-Related Genes in the Monkey Retina. J Stem Cell Res Ther 5: 319. doi:10.4172/2157-7633.1000319

Page 5 of 5

1,2-dimethylhydrazine, methylazomethanol acetate, and gamma-rays. Cancer Res 49: 6342-6346. [PubMed]

9. Cotsarelis G, Cheng SZ, Dong G, Sun TT, Lavker RM (1989) Existence of slow-cycling limbal epithelial basal cells that can be preferentially stimulated to proliferate: implications on epithelial stem cells. Cell 57: 201-209. [PubMed]

10. Sugiyama T, Katsumura K, Nakamura K, Kobayashi M, Muramatsu M, et al. (2006) Effects of chymase on the macular region in monkeys and porcine muller cells: probable involvement of chymase in the onset of idiopathic macular holes. Ophthalmic Res 38: 201-208. [PubMed]

11. Fishell G, Kriegstein AR (2003) Neurons from radial glia: the consequences of asymmetric inheritance. Curr Opin Neurobiol 13: 34-41. [PubMed]

12. Mayer EJ, Hughes EH, Carter DA, Dick AD (2003) Nestin positive cells in adult human retina and in epiretinal membranes. $\mathrm{Br} \mathrm{J}$ Ophthalmol 87: 1154-1158. [PubMed]

13. Kishi S, Hagimura N, Shimizu K (1996) The role of the premacular liquefied pocket and premacular vitreous cortex in idiopathic macular hole development Am J Ophthalmol 122: 622-628. [PubMed]

14. Maruichi M, Oku H, Takai S, Muramatsu M, Sugiyama T, et al. (2004) Measurement of activities in two different angiotensin II generating systems, chymase and angiotensin-converting enzyme, in the vitreous fluid of vitreoretinal diseases: a possible involvement of chymase in the pathogenesis of macular hole patients. Curr Eye Res 29: 321-325. [PubMed]

15. May CA (1999) Mast cell heterogeneity in the human uvea. Histochem Cell Biol 112: 381-386. [PubMed]

16. Leskinen MJ, Lindstedt KA, Wang Y, Kovanen PT (2003) Mast cell chymase induces smooth muscle cell apoptosis by a mechanism involving fibronectin degradation and disruption of focal adhesions. Arterioscler Thromb Vasc Biol 23: 238-243. [PubMed]

17. Wang Y, Shiota N, Leskinen MJ, Lindstedt KA, Kovanen PT (2001) Mast cell chymase inhibits smooth muscle cell growth and collagen expression in vitro: transforming factor-beta1- dependent and -independent effects. Arterioscler Thromb Vasc Biol 21: 1928-1933. [PubMed]

18. Leskinen M, Wang Y, Leszczynski D, Lindstedt KA, Kovanen PT (2001) Mast cell chymase induces apoptosis of vascular smooth muscle cells. Arterioscler Thromb Vasc Biol 21: 516-522. [PubMed]
19. Lazaar AL, Plotnick Ml, Kucich U, Crichton I, Lotfi S, et al. (2002) Mast cell chymase modifies cell-matrix interactions and inhibits mitogen-induced proliferation of human airway smooth muscle cells. J Immunol 169: 1014-1020. [PubMed]

20. Hara M, Matsumori A, Ono K, Kido H, Hwang MW, et al. (1999) Mast cells cause apoptosis of cardiomyocytes and proliferation of other intramyocardial cells in vitro. Circulation 100: 1443-1449. [PubMed]

21. Azuma N, Tadokoro K, Asaka A, Yamada M, Yamaguchi Y, et al. (2005) Transdifferentiation of the retinal pigment epithelia to the neural retina by transfer of the Pax6 transcriptional factor. Hum Mol Genet 14: 1059-1068. [PubMed]

22. Komitova M, Eriksson PS (2004) Sox-2 is expressed by neural progenitors and astroglia in the adult rat brain. Neurosci Lett 369: 24-27. [PubMed]

23. Fischer AJ, Reh TA (2001) Müller glia are a potential source of neura regeneration in the postnatal chicken retina. Nat Neurosci 4: 247-252. [PubMed]

24. Ooto S, Akagi T, Kageyama R, Akita J, Mandai M, et al. (2004) Potential for neural regeneration after neurotoxic injury in the adult mammalian retina. Proc Natl Acad Sci 101: 13654-13659. [PubMed]

25. Osakada F, Ooto S, Akagi T, Mandai M, Akaike A, et al. (2007) Wnt signaling promotes regeneration in the retina of adult mammals. J Neurosci 27: 42104219. [PubMed]

26. Xue L, Ding P, Xiao L, Hu M, Hu Z (2010) Nestin, a new marker, expressed in Müller cells following retinal injury. Can J Neurol Sci 37: 643-649. [PubMed]

27. Xue L, Ding P, Xiao L, Hu M, Hu Z (2011) Nestin is induced by hypoxia and is attenuated by hyperoxia in Müller glial cells in the adult rat retina. Int $\mathrm{J}$ Exp Pathol 92: 377-381. [PubMed]

28. Bhatia B, Singhal S, Lawrence JM, Khaw PT, Limb GA (2009) Distribution of Müller stem cells within the neural retina: evidence for the existence of a ciliary margin-like zone in the adult human eye. Exp Eye Res 89: 373-382. [PubMed]

29. Walcott JC, Provis JM (2003) Müller cells express the neuronal progenitor cell marker nestin in both differentiated and undifferentiated human foetal retina. Clin Experiment Ophthalmol 31: 246-249. [PubMed] 